\title{
Visual detection of signals in the presence of continuous and pulsed backgrounds'
}

BARRY LESHOWITZ, HARVEY B. TAUB, AND DAVID H. RAAB BROOKLYN COLLEGE

The detectability of intensity increments in the presence of continuous and pulsed backgrounds was investigated using a two-alternative, temporal, forced-choice procedure. Differences were found in intensity-duration reciprocity relations, the form of the Weber function, and the shape of the psychometric function between continuous- and pulsed-detection conditions. In a second study, signals were added either to a steady background (simple detection) or to background plus pedestal (pedestal detection). Two unusual phenomena were noted, namely, "negative masking" and the "pedestal effect." The interpretation of the results in terms of a simple. Poisson-detection model is discussed.

To account for the results of visual increment-detection experiments, several probabilistic models have been proposed. Examples of the quanto-statistical approach to intensity discrimination can be found in the older papers of deVries (1943), Rose (1948), Mueller (1950), and Barlow (1957), and in the more recent treatments offered by Bouman (1961), Treisman (1966), and McGill (1965). All of these formulations can be categorized as "stimulus-oriented" inasmuch as they assume that the distributions of sensory effect underlying discriminability are determined to a large extent, if not exclusively, by the Poisson statistics that describe fluctuations of light quantum emission. This type of theorizing is formally analagous to ideal-observer theory which has frequently been advanced to explain detection of auditory signals (Green \& Swets, 1966).

A review of the literature reveals that while Poisson-detection schemes in vision have not enjoyed the great success optimumdetection models have had in hearing, they have, nevertheless, been able to account for some basic psychophysical relations. The form of the Weber function, spatial summation, and temporal integration are areas in which application of the model has proved helpful (Barlow, 1957).

Although the Poisson scheme does not deal specifically with the two-alternative, temporal, forced-choice procedure, the approach can easily be generalized to include the forced-choice situation. Suppose that an increment is added to a steady background illumination in one of two nonoverlapping temporal intervals. During each observation period, which coincides with the signal's duration, it is assumed that the viewer samples a test statistic, $\Omega$, and chooses as the signal interval the interval yielding the greater value. Usually the larger statistic will be drawn from the signal-plus-background distribution, but, since all values of $\Omega$ have a finite probability under either hypothesis, there will be instances when the greater $\Omega$ will be sampled from the background-alone distribution. On these trials an incorrect decision occurs.

A basic requirement, then, for application of the signaldetection analysis is that the observer draws samples of sensory information in a similar fashion on both signal and nonsignal intervals. Moreover, in computing $\Omega$, it is assumed that the observer summates sensory information only over the area and duration of the increment. That is to say, the $S$ knows the sampling area and the observation interval exactly. It seems reasonable to conclude, therefore, that if detection theory is to serve as the model against which we wish to examine the performance of the real observer, it is essential that the experimental procedure be modified so that the observations are synchronized with the signal presentations. Classical incrementdetection experiments, in which small, brief flashes are superimposed on large continuous backgrounds during observation intervals delineated by tone pulses, hardly seem adequate to ensure that the viewer's sampling interval closely corresponds with the actual increment duration.
The aim of the present work is to study visual detection under conditions that more nearly satisfy the assumptions of detection theory. In an effort to control the S's observation period and sampling area, pedestal and pulsed-flash paradigms were employed. Experimental evidence is presented in order to assess whether psychophysical relations are a function of mode of masker presentation. In addition, conclusions are drawn concerning the adequacy of Poisson-detection schemes as models of the human visual system.

\section{EXPERIMENT 1}

In Experiment 1, the detectability of increments added either to pulsed flashes ( $p$ ulsed detection) or to continuous maskers (simple detection) was investigated using a two-alternative, temporal, forced-choice paradigm (2ATFC). The percentage of correct responses, $\mathbf{P}(\mathrm{c})$, was measured as a function of increment luminance for several levels of masker and stimulus duration.

\section{Method}

Apparatus. The light source was a glow modulator tube (Sylvania R $1131 \mathrm{C}$ ) mounted behind a ground glass plate which acted as a diffusing surface. The visual target was produced by masking off all of the ground glass plate except for a 1-cm circular aperture placed at the center of the plate. The $1-\mathrm{cm}$ target was viewed monocularly through a $1.5 \mathrm{~mm}$ artificial pupil placed 55 $\mathrm{cm}$ from the target and subtended a visual angle of $1 \mathrm{deg}$. Head position was controlled by employing a chin rest. The glow tube was bathed in ultraviolet light to reduce stimulus-onset jitter.

The target was surrounded by a ring $.75 \mathrm{~cm}$ wide and $6.3 \mathrm{~cm}$ in outer diameter. The ring served as a fixation device for the target and was illuminated from behind the S's head by a source whose intensity was adjusted so that after $5 \mathrm{~min}$ of dark adaptation the fixation ring was just visible to the S. Except for this dimly illuminated ring, the testing cubicle was completely darkened. The glow modulator tube was connected into the plate circuit of a triode-connected 6L6. A 2.7 megohm resistance was connected across the glow lamp to yield optimum switching action. In order to produce rectangular flashes, positive pulses of adjustable duration were generated by a Tektronix 162 waveform generator and delivered to the grid of the 6L6. The output of the waveform generator was split and delivered to two channels-one for $I$, the other for $\Delta I$. Each channel contained potentiometers for level setting and operational amplifiers (Philbrick K2W) for isolation. Pulses for $I$ and $\Delta I$ were mixed and then delivered to the $6 \mathrm{~L} 6$ driver.

Luminance measurements were made at the ground glass target with a calibrated vacuum phototube (RCA 934) and photopic correction filter (Kodak Wratten filter 106). The highest background level employed was $2.3 \mathrm{log} \mathrm{mL}$. In the simple-detection situation, the continuous background was produced by leaving the $6 \mathrm{~L} 6$ in a state of constant conduction. For the pulsed condition, the $6 \mathrm{~L} 6$ was biased beyond cut-off, and standard flashes were produced by overriding the bias on the triode. The current through the $6 \mathrm{~L} 6$ required to produce $2.3 \mathrm{log} \mathrm{mL}$ was approximately $40 \mathrm{~mA}$. Lower levels of luminance were produced by insertion of Kodak neutral density filters between the glow tube and the ground glass plate. Incremental-flash intensity was controlled by varying the amplitude of the positive pulse in the $\Delta I$ channel by adjusting a voltage divider. The rise and decay time of the light flash was approximately $10 \mu \mathrm{sec}$.

Luminance levels were monitored after each 15 -min session. For all conditions, it was found that the luminance levels varied over a range of $0.08 \log$ units. However, the levels of $I$ and of $\Delta I$ were 


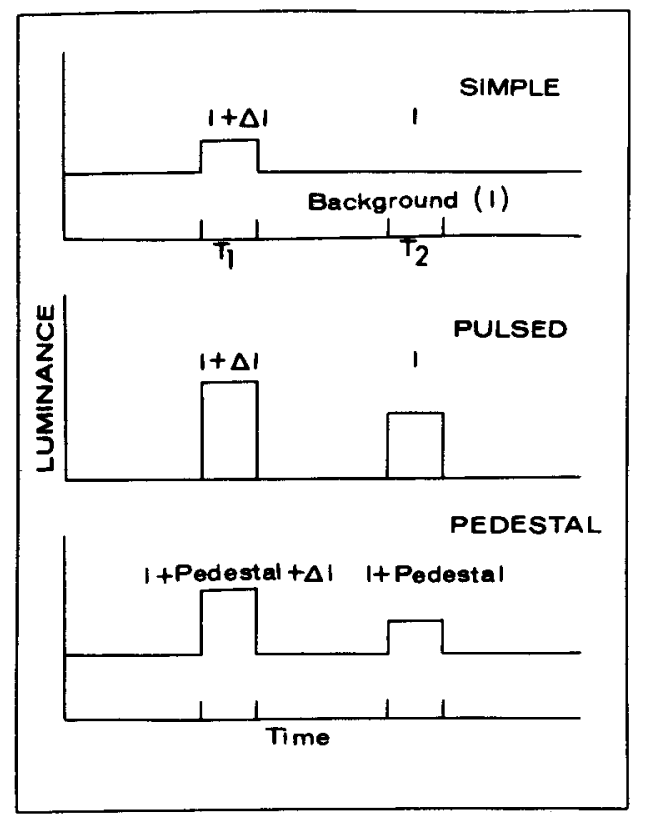

Fig. 1. Temporal sequence of stimuli within a two-alternative, temporal, forced-choice trial for the three experimental conditions. A signal, $\Delta I$, occurred in either the first or the second interval according to a random order.

found to drift together so that the predetermined values of $\Delta I / I$ were maintained.

Procedures. Preliminary experiment. It has been reported that the spectral energy distribution of light flashes derived from the glow modulator tube varies with current through the tube (Buchmann-Olsen \& Rosenflack, 1957). To determine whether such variations in the spectral distribution of irradiance accompanying the changes in current required to produce visible increments were observable, a preliminary experiment was conducted.

On each trial two $32 \mathrm{msec}$ flashes $(2.0 \log \mathrm{mL})$ were presented. On one-half of the 300 test trials the pair of flashes was produced by driving the glow tube with identical rectangular current pulses $(\approx 40 \mathrm{~mA})$. For the remaining trials, two unequal current pulses were generated so that one member of the pair of flashes measured $2.5 \log \mathrm{mL}$, and the other $2.0 \log \mathrm{mL}$. The more intense flash, which appeared randomly in either the first or second observation interval, was attenuated by inserting a $0.5 \log$ unit neutral Wratten filter (106). The task of the $S$ was to determine whether the pair of equal-luminance flashes was "the same" or "different."

The results indicated that for both Ss the probability of a correct response did not differ significantly from chance $(p=0.5)$. In view of the fact that any color changes that may have occurred in the main experiments were phenomenally undetectable, we can conclude that alterations in the spectral characteristics of the light source probably had a negligible effect on the detectability data, and, can, therefore, be ignored.

Main experiment. Figure 1 shows schematically the temporal sequence of stimulus events. Each trial in the pulsed-detection condition began with a warning click delivered to a loudspeaker. Two additional clicks spaced $0.8 \mathrm{sec}$ apart occurred $0.8 \mathrm{sec}$ after the start of the trial, and accompanied the masking flashes. Pulsed simultaneously with one or the other of the flashes was an increment whose area and duration were the same as those of the standard. The observer's task was to determine which of the two flashes had been increased in intensity. Trials were spaced $6 \mathrm{sec}$ apart.

In the simple-detection procedure, the steadily-illuminated background was briefly increased in luminance over its entire area during $T_{1}$ or $T_{2}$. We note that whereas the viewer in the pulsed-detection situation remained dark adapted throughout the session, observers in simple detection were allowed to become fully adapted to the background before presentation of the incremental flash.

Except for the type of masker-that is, continuous versus pulsed-all experimental procedures and stimulus displays employed in the two detection tasks were identical. The signal was presented in either the first or second temporal interval of the forced-choice trial according to a random order. The $S$, who was dark adapted for a minimum of 10 min before testing began, selected the interval which contained the signal by pressing one or the other of two microswitch buttons and was immediately given knowledge of results.

In each 15 -min session 50 trials were administered. A total of at least 200 observations were required to define a single value of $P(c)$, and from two to seven points were used to determine the shape and location of each psychometric function.

Subjects. Two of the authors (B.L. and H.T.) served as Ss.

Results

Data presented in Figs. 2 and 3 are representative of the results obtained in Experiment 1. Percentage of correct responses is plotted as a function of $\log (\Delta \mathrm{I} / \mathrm{I})$ for several combinations of stimulus duration and masker level for pulsed- and simpledetection conditions. Abscissa values have been scaled so that the midpoint $(\mathrm{P}(\mathrm{c})=75 \%)$ of the psychometric function for each condition corresponds to $\log (\Delta \mathrm{I} / \mathrm{I})=0$. That is, a constant has

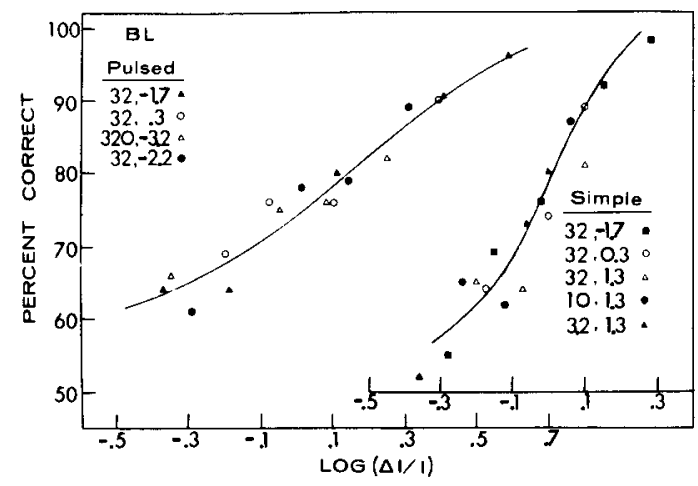

Fig. 2. Psychometric functions for simple- and pulsed-detection experiments. The percentage of correct responses in the 2ATFC task is plotted against $\log (\Delta \mathrm{I} / \mathrm{I})$. These functions were obtained at several durations and levels of masker, as indicated. by pairs of numbers which give signal duration (msec) first, and background luminance $(\log \mathrm{mL}$ ) second. The abscissa is scaled so that $\log (\Delta \mathrm{I} / \mathrm{I})=0$ corresponds to $\mathrm{P}(\mathrm{c})=75 \%$. The solid curves are the equation $d^{\prime}=c(\Delta I / I) k$, where $c$ represents an adjustment for experimental condition, and where $\mathbf{k}$ is equal to $\mathbf{2 . 0}$ and $\mathbf{0 . 7 5}$ for continuous and pulsed detection respectively. Each data point represents at least 200 observations from Observer B.L.

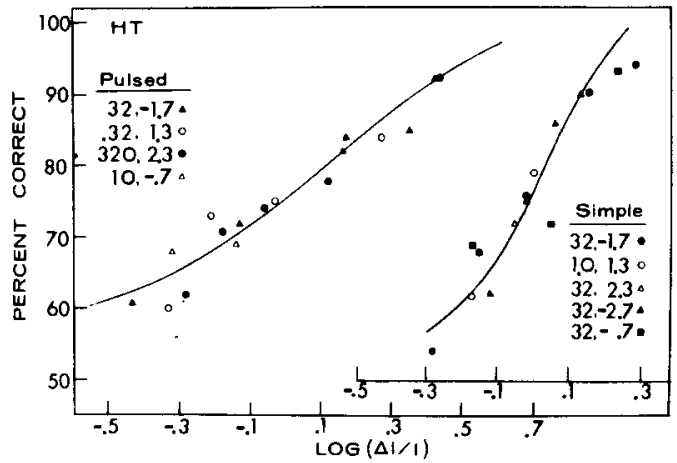

Fig. 3. Psychometric functions for Observer H.T. See legend of Fig. 2. 
been added to the scale of $\log (\Delta I / I)$ so that all psychometric functions intersect at the midpoint.

Inspection of Figs. 2 and 3 reveals that all of the data are adequately fit by psychometric functions (smooth curves) given by the relation

$$
\mathbf{d}^{\prime}=\mathbf{c}(\Delta \mathbf{l} / \mathbf{I})^{\mathbf{k}}
$$

where the constant, $c$, is a measure of the amount of masking, and $k$ provides an index of the steepness of the psychometric function. ${ }^{2}$ As can be seen from the data, the slope of the psychometric function for the pulsed case is significantly less than that observed in simple detection. To cover the range of values of $P(c)$ more than a $1.0 \mathrm{log}$ unit change in signal intensity is required for pulsed detection. For simple detection, the range is approximately $0.6 \log$ units.

Differences in the shape of the function are reflected in the values of $k$ required to produce satisfactory fits to data. Values of $\mathrm{k}$ equal to 2.0 for the simple case, and 0.75 for the pulsed, yielded acceptable fits for both Ss. Adequacy of the visual fits was corroborated by fitting curves using the method of Probit analysis (Finney, 1952). A correction was included for the truncated range of the forced-choice psychometric function. A $t$ test on the estimated slopes confirmed our earlier conclusion that continuous presentations give rise to steeper slopes than pulsed. ${ }^{3}$

Values of $\log \Delta I$ necessary for $75 \%$-correct detections were estimated from the curves fitted by eye and are plotted as constant-detectability contours in Figs. 4 and 5 . Whereas perfect temporal summation is found within the critical duration for the case of simple detection, there is a surprising absence of reciprocity between duration and signal intensity in the pulseddetection condition. That is, for the continuous masker condition, it was found that $\Delta I X T=K$ for durations less than $32 \mathrm{msec}$, and for the pulsed case $\Delta I=K$. Only when the pulsed masking flash approaches absolute threshold do we notice any decrement in performance as signal duration is shortened.

Not only is the absolute level of performance unaffected by changes in stimulus duration, but also the shape of the psychometric function appears to remain invariant in the face of a more than 3.5-log-unit change in flash duration (see Fig. 6). For S B.L., a single psychometric function adequately describes $P(c)$ as a function of $\log (\Delta I / I)$. Note that no "abscissa shift" has been applied to these data. To account for results obtained with less intense pulsed maskers (Observer H.T.), however, two psychometric functions of the same shape are required.

Plots of $\Delta \mathrm{I} / \mathrm{I}$ against $\log 1$ for 32 - and 320-msec-pulsed conditions and for simple detection of 32-msec increments are presented in Figs. 7 and 8. The data show that the unusual Weber

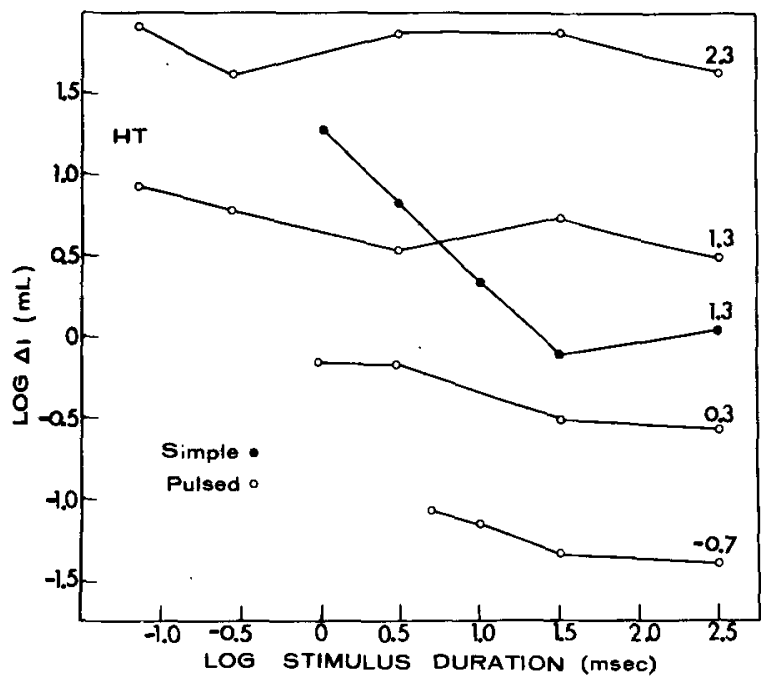

Fig. 4. $\log \Delta \mathrm{d}(\mathrm{mL})$ necessary for $75 \%$-correct detections as a function of stimulus duration for Observer B.L. for pulsed and simple detection. The parameter is the level of the background $(\log \mathrm{mL})$. functions reported by Cornsweet and Pinsker (1965) were obtained in the present study. For pulsed detection, we observe a small departure from a constant Weber fraction at low luminance levels. Contrast this result with the classical Weber function found in simple detection, where $\Delta I / I$ displays a marked increase as the background illumination is made weaker (Herrick, 1956).

Replotting the masking relations appearing in Figs. 7 and 8 in terms of $\log \Delta \mathrm{I}$ and $\log \mathrm{I}$ permits several interesting observations (see Figs. 9 and 10), On the one hand, a linear relationship between $\Delta I$ and $I$ obtains in the pulsed case throughout almost the entire range of 1 ; on the other hand, simple detection gives rise to a function that is more nearly described by a square root law of the form: $\Delta I=\mathrm{KI}^{1 / 2}$. We note that the function for simple detection intersects both curves for the pulsed case. At high masking luminances, signals are more easily detected in the presence of continuous maskers than when added to gated flashes. The reverse is true at low levels. Apparently, then, the relative masking effectiveness of continuous and pulsed backgrounds is level-dependent.

\section{EXPERIMENT 2}

In Experiment 2 two studies were conducted using a pedestaldetection paradigm. In addition to a steady background, a pulsed flash (pedestal), whose duration and area were the same as those of the signal to be detected, was presented in each observation interval. And as was the case in simple detection, the signal covered the same area as the background (see Fig. 1). The S's task was to identify on which pedestal the signal was placed.

\section{Method 1}

In the first study incremental flashes were added to $-1.7-\log -\mathrm{mL}$ pedestals in the presence of a $-1.7-\log -\mathrm{mL}$ background, and the proportion of correct responses was measured as a function of signal intensity using a $2 \mathrm{ATFC}$ procedure.

\section{Results 1}

Psychometric functions obtained for simple and pedestal conditions shown in Figs. 11 and 12 reveal two unusual phenomena: (1) the pedestal effect-that is, the change in range of the psychometric function from $0.6 \mathrm{log}$ units in the no-pedestal or simple condition to more than $1.0 \mathrm{log}$ units when the signal to be detected is an increment added to a pedestal in the presence of an ongoing masker; (2) negative masking, the term used to describe the result wherein Ss can more easily detect a signal added to pedestal-plus-background than when added to background alone.

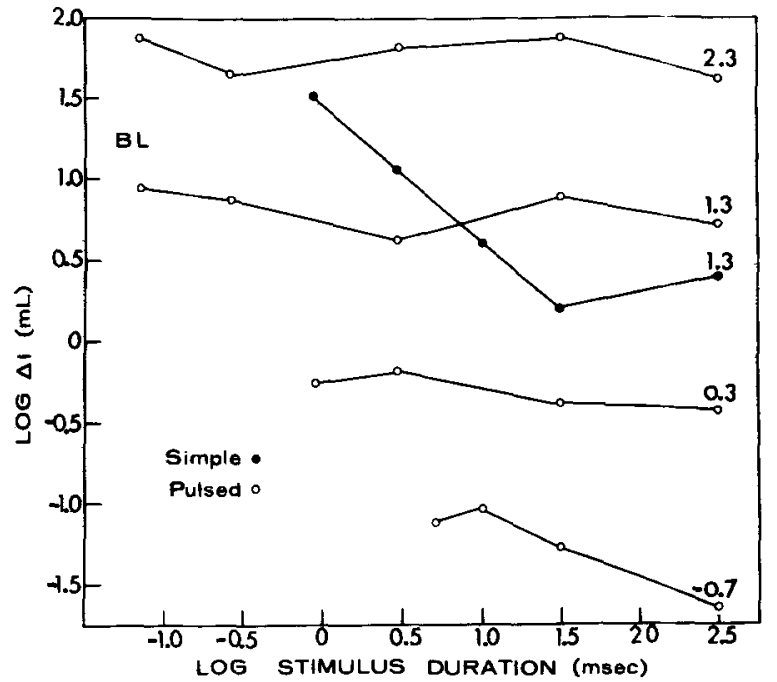

Fig. 5. Constant detectability contours for Observer H.T. See legend of Fig. 4. 


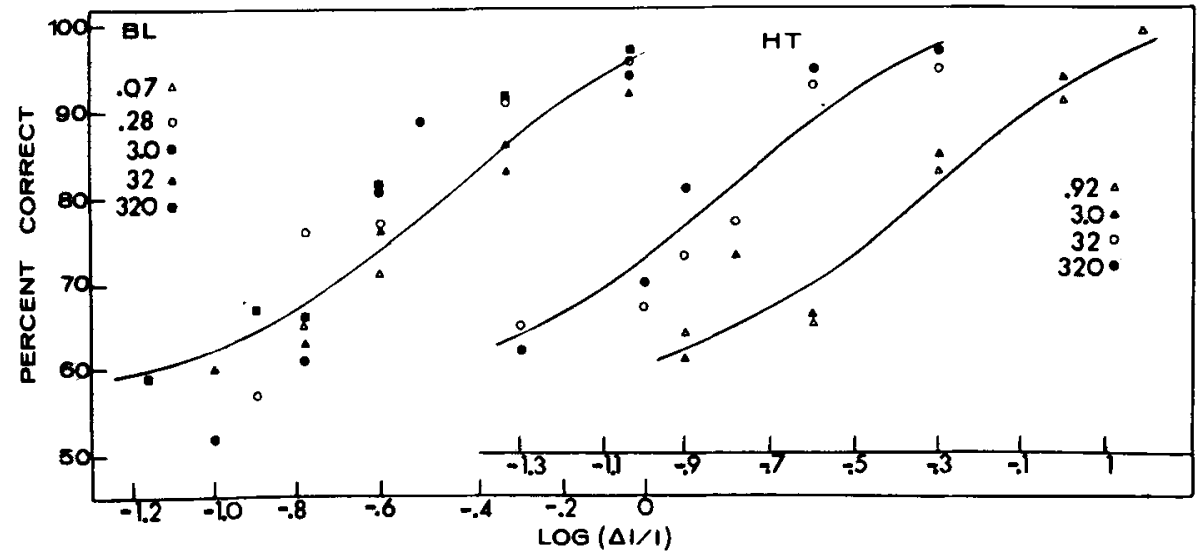

Fig. 6. Pychometric functions for pulad detection for Observers B.L. and H.T. These functions were obtuined at several durntions (mece), as indicated, and with maker levels equal to $2.3 \log \mathrm{mL}$ (Oberver B.L.) and $0.3 \mathrm{log} \mathrm{mL}$ (Observer H.T.).

A measure of the amount of negative masking is given by the reduction in the absolute magnitude of $\Delta I$ necessary for $75 \%$ correct detections. For both observers, the difference in the detection thresholds was approximately $0.4 \mathrm{log}$ units.

\section{Method 2}

In view of the fact that "negative masking" is a topic still in dispute in both vision and hearing (see e.g., Green, 1966; Leshowitz \& Raab, 1967; Nachmias, 1966), it was decided to conduct a second experiment employing pedestals.

The background $(-1.7 \log \mathrm{mL})$ as well as the signal $(-2.0 \log \mathrm{mL})$ were held constant in this study, and changes in $P(c)$ were recorded as the pedestal level was varied.

\section{Results 2}

Results of this second pedestal experiment are displayed in Fig. 13 , where $P(c)$ is plotted as a function of pedestal luminance. Observe that for a constant ratio of signal-to-background intensity, detectability improves with pedestal amplitude until a peak is reached, after which performance declines. Data collected in Experiment 2 thus provide strong evidence supporting the assertion that in addition to changing the slope of the psychometric function, the presence of a pedestal improves detectability.

\section{DISCUSSION}

That different discriminatory processes underlie detection of visual signals in the presence of steady and pulsed backgrounds has

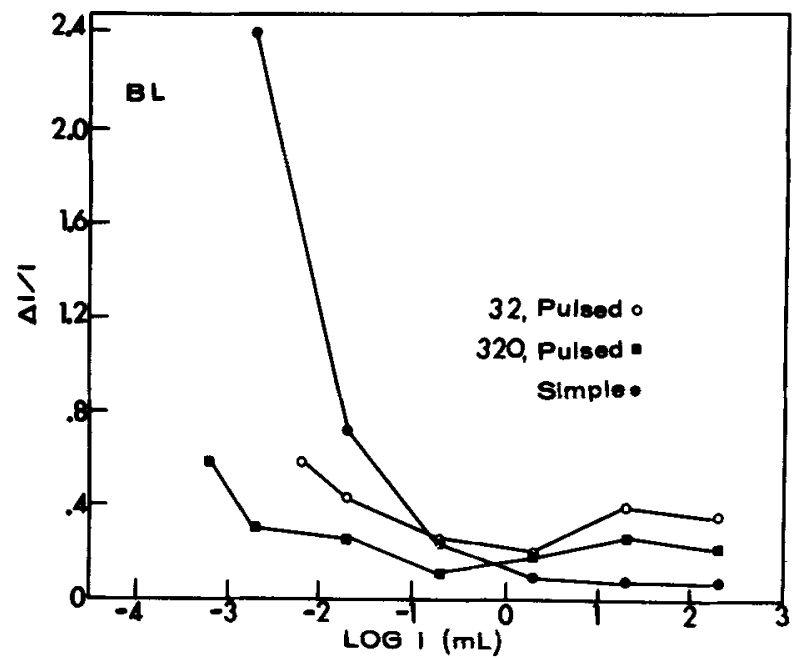

Fis. 7. Weber functions for pulsed detection for 32- and 320-msec signals and for imple detection of a 32-msec increment. Data for Observer B.L. been suggested by Bartlett (1942) and Cornsweet and Pinsker (1965) in discussions of the atypical Weber function that characterizes luminance discrimination of brief flashes under complete dark adaptation. Blackwell (1963), in his critique of the theory of signal detectability, has focused most clearly on the importance of considering experimental procedures and stimulus displays in any theoretical analysis of visual masking. According to Blackwell, observers compare samples of sensory information only in the stimulus-comparison experiment wherein the task of the viewer is to judge whether an inner disk is brighter or darker than a concentric ring. Essentially, Blackwell's objection to the signaldetectability analysis of the forced-choice task is that it has been incorrectly applied to the stimulus-increment or simple detection situation.

Consistent with Blackwell's criticisms are the data obtained in Experiment 1, where it was found that there are important differences in several fundamental psychophysical relations between continuous and pulsed conditions. The slope of the psychometric function, the form of the Weber function, intensityduration reciprocity, and the dependence of relative masking effectiveness on overall level were all shown to be a function of mode of masker presentation. These data suggest that we are probably not entitled to the simplifying assumptions frequently made by signal-detection theorists, namely that the viewer computes differences and knows the signal duration and area exactly regardless of the type of masker employed.

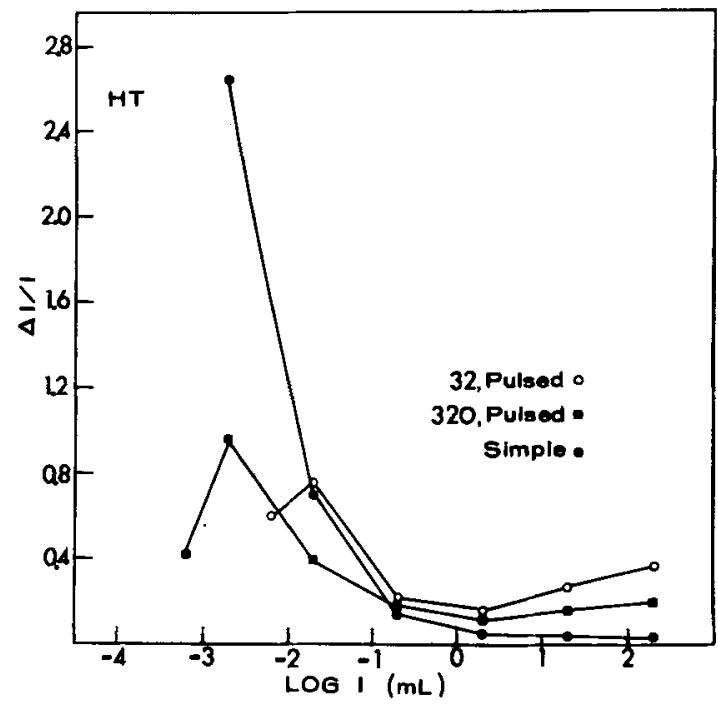

Fig. 8. Weber functions for Observer H.T. See legend for Fig. 7. 


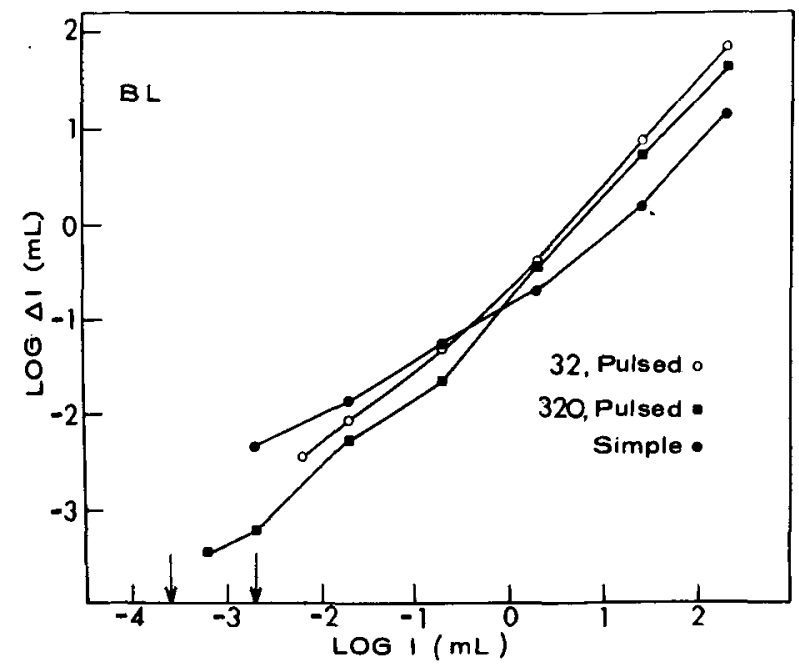

Fig. 9. $\log \Delta I$ plotted as a function of the luminance of the masker $(\log I)$. Data for pulsed detection of 32- and 320-msec signals, and for simple detection of 32-msec signals. The arrows at -2.7 and $-3.6 \log \mathrm{mL}$ are the absolute thresholds for the 32- and 320-msec pulsed flashes. Data for Observer B.L.

\section{Continuous-Pulsed Differences in Audition}

To the extent that the continuous-pulsed differences obtained in vision reflect general properties of sensory systems, we ought to find a similar situation in audition. In agreement with this hypothesis are data obtained by Dirks and Norris (1966) who tracked the variation of tonal signal power required for $75 \%$ correct detections as a function of noise level for continuous and pulsed cases. Throughout most of the intensity range, gated noise produced approximately $5 \mathrm{~dB}$ more masking than steady noise. However, as the level of the masker approached absolute threshold, this difference in masking effectiveness between pulsed and continuous conditions ceased to exist. For the case of tones added to tones of identical frequency or to a complex masker consisting of three sinusoids, Campbell and Lasky (1967) have also shown that pulsed stimuli are more effective maskers than continuous stimuli and that the difference in masking effectiveness disappears at low levels. They reported that for short-duration signals (e.g., $20 \mathrm{msec}$ ) there is a reversal of the Weber function at low masker levels with the gated presentation yielding lower signal-to-masker ratios than the continuous.

Effects of duration on detection of tonal signals with continuous and gated noise are also similar to the relations observed in vision. Tucker, Evans, and Jeffress (1966) found that when signal energy is held constant, gated-noise-and-signal conditions at long duration $(500 \mathrm{msec})$ yields $\mathrm{d}$ 's that are slightly worse than those obtained with continuous maskers, though the reverse is true at short durations.

Unfortunately, not all of the auditory temporal integration data are consonant with the continuous-pulsed differences observed in vision. Wightman and Green (1966) have recently studied the effects of duration on detection of noise increments added to gated noise bursts. Whereas in Experiment 1 we found that with luminous power of the standard flash held constant no improvement in performance obtained with increases in duration, Wightman and Green measured a reciprocity factor of $4 \mathrm{~dB}$ per ten-fold change in duration.

Other psychoacoustic data incompatible with the present set of vision results come from studies of the shape of the psychometric function. Green (personal communication) has stated that the difference in the slope of the psychometric function between gated and continuous conditions for detection of noise and sinusoidal signals masked by band-limited noise appears to be much less than what obtains in vision. Differences between gated and continuous psychometric functions have been noted by
Campbell and Lasky (1967) for the case of tone added to tonc. The authors found that for 20-msec signals, gated masker conditions give substantially lower slopes than continuous. With 400-msec signals, however, slopes of gated and continuous conditions were identical.

It is interesting to note that while investigators in hearing have not observed any consistent deviations in the form of the psychometric function for pulsed maskers, dramatic changes in the slope have been recorded when signals are added to gated maskers in the presence of an ongoing background noise (Green, 1960; Green \& Sewell, 1962). This finding has been termed the "auditory pedestal effect" and is similar to the visual pedestal effect obtained in Experiment 2.

\section{Theory of Ideal Observers}

The decrease in slope of the psychometric function observed when a sinusoidal signal is an in-phase addition to a gated sinusoid of the same duration and frequency has been offered as evidence in support of the correlation-detector-for-the-case-ofthe-signal-known-exactly as an appropriate analog of the human auditory system (Green, 1960). Whereas in the simple or pedestalless condition it was found that the obtained psychometric function is considerably steeper than the theoretical, with the addition of an attenuation constant, the correlation detector accurately predicted $\mathbf{P}(\mathrm{c})$ for pedestal detection. To explain these data, Green has proposed a "memory hypothesis" which posits that the function of the gated pedestal is to provide the listener with information regarding frequency, phase, time of occurrence, and duration of the signal. Effective reduction of observer uncertainty is, according to Green, reflected as a decrease in the slope of the psychometric function.

The notion of ideal sensor systems has been generalized to the area of visual signal detection (Tanner \& Jones, 1959). According to the theory, the shape of the psychometric function in vision reflects observer uncertainty with respect to time of onset, duration, and retinal location of the signal. Moreover, as the degree of uncertainty increases, the theory posits that performance deteriorates, with weak signals being more adversely affected than strong signals. Communication engineers refer to the relation of increasing slope to decreasing sensitivity as "weak signal suppression." As can be seen in Figs. 11 and 12, the addition of an information-carrying pedestal produced both effects-that is, enhanced detectability and a significant flattening of the psychometric function. These findings lend credence to the conclusion first advanced by Green and Sewell (1961) in audition that gated pedestals do serve to reduce temporal uncertainty, thus rendering human behavior more "optimum." An alternative explanation of the pedestal data will be considered later.

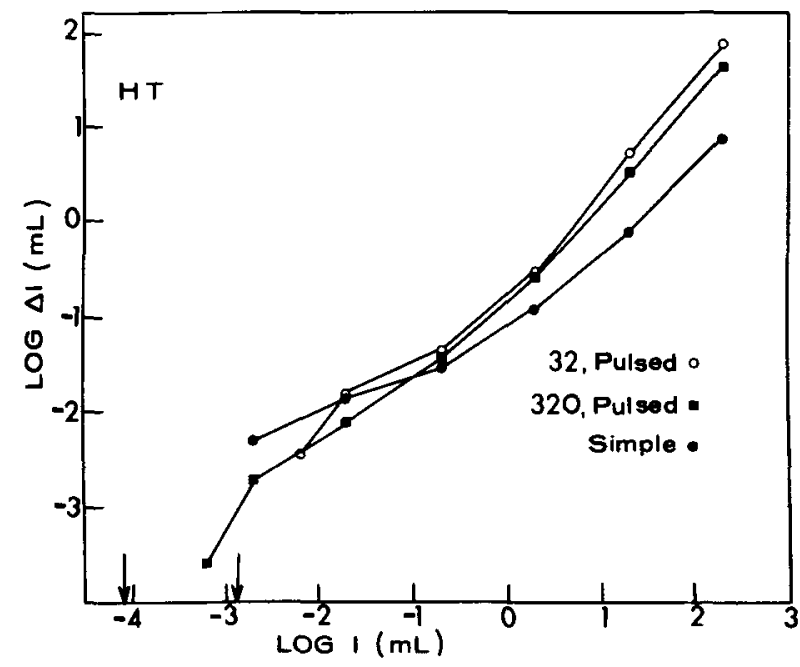

Fig. 10. Masking relations for Observer H.T. See legend for Fig. 9. 


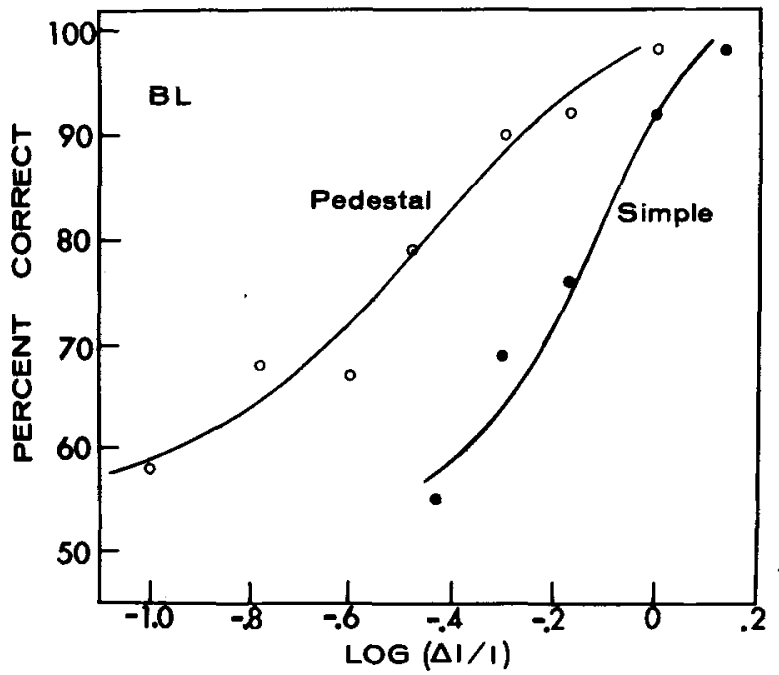

Fig. 11. Psychometric functions for simple- and pedestal-detection experiments. The steady background and pedestal were both equal to $-1.7 \mathrm{log}$ $\mathrm{mL}$, and the duration of the signal was $32 \mathrm{msec}$. The solid curves are the equation $d^{\prime}=c(\Delta I / I)^{k}$, where $c$ represents an adjustment for experimental condition, and where $k$ is equal to 2.0 and 1.05 for continuous and pedestal detection respectively. Each data point represents 300 observations from Observer B.L.

It has been demonstrated in hearing, and now in vision, that the range of the psychometric function changes systematically as the pedestal level is varied, but there are no auditory data that display negative masking. Monaural addition of stimuli to the auditory system has been shown to produce only positive masking. Moreover, a search of the literature on intensity discrimination failed to uncover the existence of negative masking in vision. Thus, the present set of pedestal data constitutes the only evidence of "release from masking" obtained to date in either vision or hearing.

Comparison of the Poisson-Detection Model and Human Observers

Coupled with the intensity-duration trades observed in the pulsed-detection task, the facilitating effects of pedestals obtained in Experiment 2 cause great difficulties for a Poisson scheme. Recall that the theory assumes that the distributions of sensory

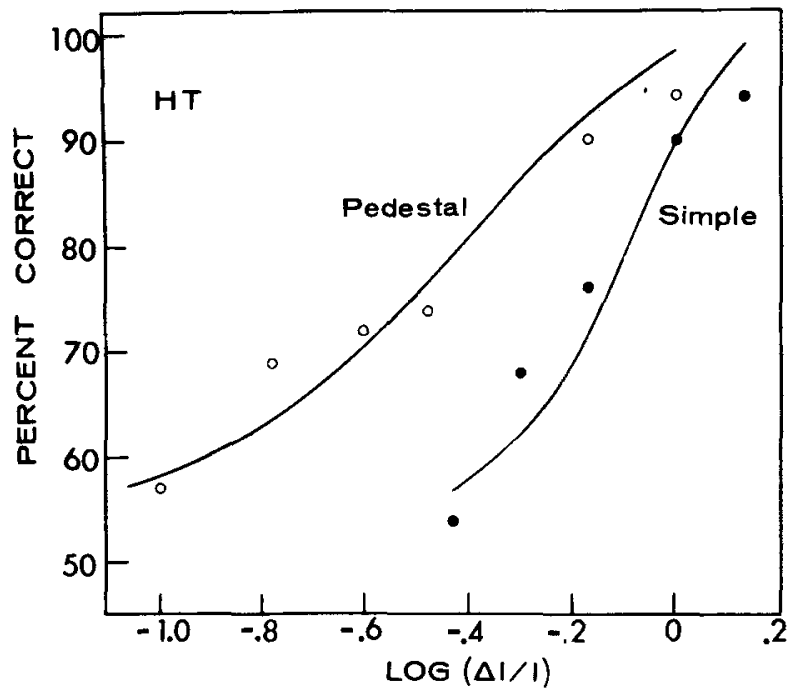

Fig. 12. Psychometric functions for simple and pedestal detection for Observer H.T. See legend for Fig. 11. effect are shaped by the Poisson statistics governing fluctuations of light quantum emission. Since the mean of the Poisson distribution is equal to its variance, variability of sensory information is tied to the expected value of the process. Hence, increasing the duration of a flash causes a greater number of light quanta to be absorbed and a concomitant increase in the ratio of the mean to sigma of the sampled test statistic. What is important, then, about increasing flash duration is the improvement in the tolerance of measurement. More reliable estimates of the average energy ought to give rise to smaller masked thresholds. As is seen in Figs. 4 and 5, extending the light pulse in time does not yield better discriminability.

Enhanced detectability, noted in pedestal detection, is similarly incompatible with a Poisson model. Nachmias (1966), commenting on Baumgardt and Smith's (1965) finding that a continuously presented subthreshold background sometimes reduces the absolute threshold, has pointed out that introduction of a background stimulus can only increase the variability of sampled "counts," and would leave the mean difference unaffected. Hence, detectability should be worse, not better, in the presence of an additional masking source. If Nachmias' arguments are applied to the visual pedestal data, it appears that we are forced to abandon the Poisson scheme.

A signal-detection approach may be salvaged if we assume that the phenomenon of negative masking is merely a consequence of the nonlinear relation between $\mathrm{d}^{\prime}$ and $\Delta \mathrm{I} / \mathrm{I}$. Let us define for the pedestal detection case the quantity

$$
I_{p+s}=I_{s}+I_{p} \text {, }
$$

where the ratio of signal-to-background intensity is $I_{S}$, and $I_{p}$ is pedestal/background. Recall that the fitted psychometric function for simple detection was based on the general relationship:

$$
\mathrm{d}^{\prime}=\mathrm{I}_{\mathrm{p}}^{2} \mathrm{~s} \text {, }
$$

Thus, $d^{\prime}$ for signals detected in the presence of pedestals is given by

$$
\begin{aligned}
& d_{p}^{\prime}=d^{\prime}\left(I_{p+s}\right)-d^{\prime}\left(I_{p}\right)=I_{p+s}^{2}-I_{p}^{2}, \\
& d_{p}^{\prime}=I_{s}^{2}+2 I_{s} I_{p} .
\end{aligned}
$$

Consider now values of $\mathrm{d}^{\prime}$ that result from the addition of a signal to noise alone and to noise-plus-pedestal. On the assumption that $d^{\prime}$ grows as a positively accelerating function of $I p+s$ over the range of stimulus values used in Experiment 2, a weak signal added to noise may be detected near chance, while the same signal added to a small pedestal may be perfectly detectable.

We also note that the above account is completely consistent with the present pedestaleffect result. When $I_{S}<2 I_{p}$, it follows from Equation (3) that $d_{p}^{\prime} \approx K_{s}$. We would predict, therefore,

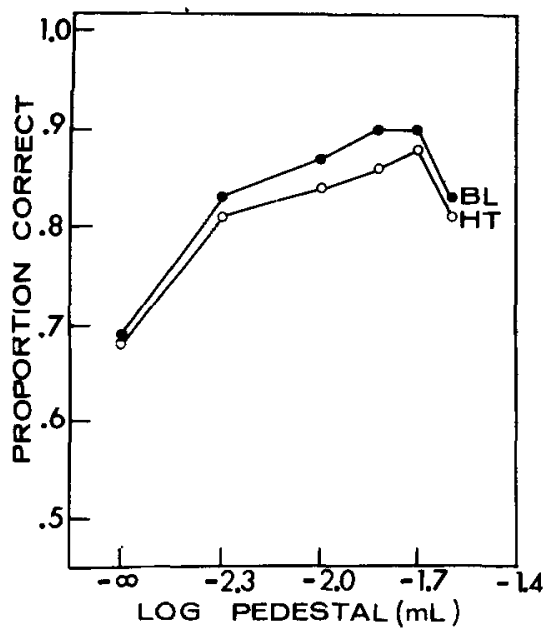

Fig. 13. Proportion of correct responses as a function of pedestal level with signal/background constant. The continuous background was $-1.7 \mathrm{log}$ $\mathrm{mL}$. Signal luminance was equal to $-2.0 \mathrm{log} \mathrm{mL}$. Each data point represents 300 observations from Observers B.L. and H.T. 
that whereas the psychometric function in the simple detection case would show that $d^{\prime}$ varies with the square of signal strength, the psychometric function for pedestal detection would show $\mathrm{d}^{\prime}$ to be a linear function of $\Delta \mathrm{I} / \mathrm{I}$. Inspection of Figs. 11 and 12 reveals just this result.

\section{CONCLUDING REMARKS}

The serious discrepancies between the Poisson model and data forces the conclusion that the theory in its present form has serious limitations and must be extensively modified. In an attempt to unify stimulus- and organism-oriented approaches to visual detection, Treisman (1966) has proposed a neurophysiological model that takes into account the statistics of light and the variability of neural messages in sensory channels. The Treisman model can be extended to the 2ATFC-continuous- and pulsedmasker situations. It can be shown that the model is easily able to generate many of the familiar intensity-duration and masking relations that characterize visual detection in the presence of continuous and pulsed maskers. The success of Treisman's statistical decision model, together with the apparent failure of the exclusively stimulus-oriented approach, strongly suggests that if theories of detection are to be useful, they must incorporate the statistics of the stimulus and the flow of information in sensory channels.

\section{REFERENCES}

BARLOW, H. B. Increment thresholds at low intensities considered as signal/noise discrimination. J. Physiol., 1957, 136, 469-488.

BARTLETT, N. R. The discrimination of two simultaneously presented brightnesses. J. exp. Psychol., 1942, 31, 380-392.

BAUMGARDT, E., \& SMITH, S. A. Facilitation effect of background light on target detection. Vision Res., 1965, 5, 299-312.

BLACKWELL, H. R. Neural theories of simple visual discrimination. J. Opt. Soc. Amer., 1963, 53, 129-160.

BOUMAN, M. A. History and present status of quantum theory in vision. In W. A. Rosenblith (Ed.), Sensory communication. Cambridge, Mass.: MIT Press, 1961. Chap. 21.

BUCHMAN-OLSEN, B., \& ROSENFLACK, A. M. Spectral energy calibration of a light flash source used in physiological experiments. J. Opt. Soc. Amer., 1957, 47, 30-34.

CAMPBELL, R. A., \& LASKEY, E. Z. Masker level and sinusoidal-signal detection. J. Acoust. Soc. Amer., 1967, 42, 972-976.

CORNSWEET, T. N., \& PINSKER, H. M. Luminance discrimination of brief flashes under various conditions of adaptation. J. Physiol., 1965, 176, 294-310.

DIRKS, D. D., \& NORRIS, J. C. Shifts in auditory thresholds produced by ipsilateral and contralateral maskers at low-intensity levels. $J$. Acoust. Soc. Amer., 1966, 40, 12-19.

EGAN, J. P. Masking-level differences as a function of interaural disparities in intensity of signal and of noise. J. Acoust. Soc. Amer., 1965, 38, 1043-1049.

ELLIOT, P. B. Tables of $\mathrm{d}^{\prime}$. In J. A. Swets (Ed.), Signal detection and recognition by human observers. New York: Wiley, 1964. Pp. 651-684.

FINNEY, D. J. Probit analysis. Cambridge, England: Cambridge University Press, 1952.

GREEN, D. M. Psychoacoustics and detection theory. J. Acoust. Soc. Amer., $1960,32,1189-1203$.

GREEN, D. M. Comments on: Effects of waveform correlation and signal duration on detection of noise bursts in continuous noise. [D. H. Raab, $E$. Osman, and E. Rich, J. Acoust. Soc. Amer., 35, 1942-1946 (1963)].J. Acoust. Soc. Amer., 1966, 39, 748-749.
GREEN, D. M., \& SEWELL, S. T. Effect of background noise on auditory detection of noise bursts. J. Acoust. Soc. Amer., 1962, 34, 1207.1216.

GREEN, D. M., \& SWETS, J. A. Signal detection theory and psychophysics. New York: John Wiley and Sons, 1966.

HERRICK, R. M. Foveal luminance discrimination as a function of the duration of the decrement or increment in luminance. J. comp. physiol. Psychol, 1956, 49, 437-443.

LESHOWITZ, B., \& RAAB, D. H. Effects of stimulus duration on the detection of sinusoids added to continuous pedestals. $J$. Acoust. Soc. Amer., 1967, 41, 489-496.

McGILL, W. J. Poisson counting and detection in sensory systems. Unpublished manuscript, University of California, San Diego, 1965.

MUELLER, C. G. Quantum concepts in visual intensity-discrimination. Amer. J. Psychol, 1950, 63, 92-100.

NACHMIAS, J. "Photosensitization" and intrinsic noise in the visual system. Vision Res, 1966, 6, 113-115.

ROSE, A. The sensitivity performance of the human eye on the absolute scale. J. Opt. Soc. Amer., 1948, 38, 196-208.

TANNER, W. P., Jr., \& JONES, R. C. The ideal sensor system as approached through statistical decision theory and the theory of signal detectability. In Proceedings of the Armed-Forces-NRC Vision Committee, held in Washington, D.C., November, 1959.

TREISMAN, M. A statistical decision model for sensory discrimination which predicts Weber's law and other sensory laws: Some results of a computer similation. Percept. \& Psychophys., 1966, 1, 203-230.

TUCKER, A., EVANS, R. B., \& JEFFRESS, L. A. Effect of duration upon detection with gated and continuous noise and signal. J. Acoust. Soc. Amer., 1966, 40, 1250.

de VRIES, H. T. The quantum character of light and its bearing upon the threshold of vision, the differential sensitivity and acuity of the eye. Physica, 1943, 10, 553-564.

WIGHTMAN, F. L., \& GREEN, D. M. Effect of duration on detection of a noise signal. J. Acoust. Soc. Amer., 1966, 40, 1250.

\section{NOTES}

1. This investigation was supported in part by a United States Public Health Service predoctoral fellowship (awarded to B.L.), by grants from the National Science Foundation, and by funds supplied by Brooklyn College.

2. Psychometric functions were fitted by transforming empirical estimates of $\mathrm{P}(\mathrm{c})$ to values of $\mathrm{d}^{\prime}$ by referring to tables provided by Elliot (1964). Straight lines were then visually fitted to plots of $\mathrm{d}^{\prime}$ against $\Delta \mathrm{I} / \mathrm{I}$ on logarithmic coordinates, and the best fitting values of $\mathrm{c}$ and $\mathrm{k}$ were selected. Since Elliot's tables assume Gaussian density functions of equal variance, $P(c)$ is a Gaussian function of $\mathrm{d}^{\prime}$. In the region where $\mathrm{k}$ is not too different from unity, the monotonic relation between $\Delta \mathrm{l} / \mathrm{I}$ and $\mathrm{d}^{\prime}$ assumed by the fitted equation gives rise to a psychometric function that closely resembles the cumulative Gaussian function. It is important to note that the two-alternative forced-choice procedure produces a psychometric function that starts at $P(c)=50 \%$. This function is, therefore, only the upper half of the ogive. The lower "tail" of the curve arises when $\Delta \mathrm{I} / \mathrm{l}$ is plotted on a log scale. A similar fitting procedure has been employed by Egan (1965) in connection with auditory masking-level differences.

3. The authors are grateful to Dr. Harry Levitt for providing us with the programs for the Probit analysis and making available to us the computer facilities at Bell Telephone Laboratories.

4. The authors are indebted to Dr. Jacob Nachmias who suggested this explanation of both negative masking and the pedestal effect.

(Accepted for publication June 1, 1968.) 